\title{
Do pregnancy rates differ with intra-uterine insemination when different combinations of semen analysis parameters are abnormal?
}

\author{
(D) Anita Kuriya1, (D) Chioma Agbo22, (D) Michael H. Dahan¹ \\ 1Division of Reproductive Endocrinology and Infertility, Department of Obstetrics and Gynecology, McGill University Health \\ Center, Montreal, Canada \\ 2Department of Emergency Medicine, Stanford University School of Medicine, California, USA
}

\section{Abstract}

Objective: To evaluate the relationship of one or a combination of semen analysis parameter results on insemination outcomes.

Material and Methods: A retrospective analysis was performed to evaluate the effect on pregnancy rates in relation to one or more abnormal semen analysis parameters based on the 2010 World Health Organization semen analysis guidelines.

Results: Nine hundred eighty-one couples underwent 2231 intrauterine insemination cycles at the Stanford Fertility and Reproductive Medicine Center. In our study, the pregnancy rates ranged from 11-25\% when an individual or combined semen analysis parameters were analyzed. Similar pregnancy rates were found when one, two, and in most cases three parameters were abnormal. When a single parameter was abnormal among volume, concentration, and motility, pregnancy rates were mainly unaffected. There was the exception of total sperm count where pregnancy rates were diminished when counts were below 39 million $(p=0.04)$.

Conclusion: Clearly, total sperm in the specimen and not the concentration of sperm per milliliter was the critical factor for predicting pregnancy. Therefore, a reorganization of semen analysis reports should be done emphasizing the total amount of sperm present and de-emphasizing concentration of sperm. (J Turk Ger Gynecol Assoc 2018; 19: 57-64)

Keywords: Artificial insemination, pregnancy rate, semen analysis

Received: 21 July, 2017 Accepted: 16 March, 2018

\section{Introduction}

Infertility is the failure to conceive following twelve months of unprotected intercourse (1). Studies suggest that infertility affects 10 to $15 \%$ of the reproductive population (1). Male factor infertility is responsible for up to $50 \%$ of infertility cases (1). Male factor infertility is diagnosed primarily based on the results of at least two semen analyses performed 90 days apart. A semen analysis consists of a wide range of parameters including: volume, sperm concentration, progressive motility, and morphology. The total motile sperm count (TMSC) is calculated by multiplying the total sperm in the specimen by the percentage of motile sperm and is felt to be an essential predictor of intrauterine insemination (IUI) success (2).
When faced with severe male factor infertility, although there exists a lack of randomized control trials, the consensus is to offer in vitro fertilization (IVF) with intracytoplasmic sperm injection (ICSI) (3). Others argue that IVF should not be considered for routine use (3) and question its cost-effectiveness for most cases of male factor infertility (3). In these cases, it is argued that IUI should be the first-line treatment instead $(3,4)$.

Most male partners of couples presenting for infertility will have one or more abnormal parameters in their semen analysis. Many have studied the effect of single parameters in relation to pregnancy and fertilization outcomes (5-8), or combining some of these parameters into the TMSC (9-12). However, there exists a lack of literature on whether a combination of parameters or any specific parameter (except for TMSC) would allow for 
lower pregnancy rates with IUI. Therefore, the objective of this study was to evaluate the effect on pregnancy rates with one or more abnormal semen analysis parameters based on the 2010 World Health Organization (WHO) semen analysis guidelines.

\section{Material and Methods}

A retrospective analysis was performed on 2.5 years of data collected at an American University. A total of 981 couples underwent 2231 IUI cycles. The original database contained information regarding evaluation of semen quality on the day of insemination. Subjects at the clinic are 40\% Caucasian, 7\% African American, 33\% Asian, and 20\% Hispanic. The biochemical pregnancy rate was 14\%, the clinical pregnancy rate was $82 \%$, and the ectopic pregnancy rate was $4 \%$.

Semen quality was classified on the day of insemination based on the 2010 WHO semen criteria. Criteria used were $1.5 \mathrm{~mL}$, 15 million/mL, minimum count per specimen 39 million, and forward motility $32 \%$. If Kruger strict morphology was less than $4 \%$ in two samples, the patients were treated with IVF and ICSI. These patients were not included in this study. It should also be noted that strict morphology is not traditionally calculated on each specimen being used for insemination because preparation would kill some of the sample required for use, possibly affecting pregnancy rates. It should also be noted that total motility was not calculated by the computer semen analyzer and was therefore unavailable for comparison. The 2010 WHO criteria also list a minimum total motility of $40 \%$ in the specimen as criteria of normality. However, forward motility of $32 \%$ was used because this and not total percent motility was available in the processing report. The specimens were compared based on the presence of all criteria being normal or one or more being abnormal.

The evaluation and examination of patients: The couples enrolled in this study had at least one year of either primary or secondary infertility with their current partner. All couples underwent a comprehensive evaluation including medical history and physical examination, documentation of ovulation or an assessment for the lack thereof, as well as a semen analysis using Kruger strict morphology. All patients had at least one patent fallopian tube on either hysterosalpingogram or laparoscopy with chromopertubation. Ovulation was evaluated with a luteal phase progesterone $>3 \mathrm{ng} / \mathrm{mL}$, basal body temperature charts, urinary luteinizing hormone (LH) kits with regular cycles every 21 to 35 days, or regular periods every 21 to 35 days with a clear history of premenstrual molimina. All women had serum prolactin and thyroid-stimulating hormone levels in the normal range of the assay used before starting treatment. Women were included if they were anovulatory with inducible ovulation, if they had a serum follicle-stimulating hormone levels $<12$ IU/L on basal and clomiphene citrate challenge testing (if performed), a baseline follicle count of greater than 8 on endovaginal ultrasonography or stage 1-2 endometriosis on laparoscopy with at least one patent and undamaged fallopian tube. All women were evaluated with hysterosalpingography or hysteroscopy, and any intra-cavitary pathology including polyps, fibroids, and synechiae were corrected before initiating treatment. Any patients with four or more myometrial fibroids of $1 \mathrm{~cm}$ or greater in diameter, or one leiomyoma of $5 \mathrm{~cm}$ or greater in the uterine muscle, underwent surgical resection and appropriate recovery before initiating the insemination cycle.

Couples did not have, women with bilaterally blocked fallopian tubes, decreased ovarian reserve, stage 3 or 4 endometriosis, recurrent pregnancy loss (2 or more miscarriages), two previous ectopic pregnancies or anovulation and folliculogenesis was not successfully induced. Donor frozen IUI semen results were excluded because only post-processing parameters were available for these samples, and the donor was unlikely to be infertile. Only partners' fresh sperm specimens were included in the analyses.

Seven percent of patients were treated with natural cycle IUI, $54 \%$ were treated with clomiphene IUI, 3\% were treated with letrozole IUI, and $36 \%$ received gonadotropin IUI. Gonadotropin injections were performed daily starting on cycle day 2 or 3 and titrated to develop 2 to 3 mature follicles in patients aged under 40 years, and 2 to 5 follicles in women aged over 40 years. Clomiphene citrate (50 or $100 \mathrm{mg}$ daily) and letrozole (5 mg daily) were administered orally for five days starting on cycle day 2 to 4 . Serial sonography was performed to monitor folliculogenesis as per standard protocols.

Semen collection, analysis, and processing: Individuals were asked to refrain from ejaculation for two to four days before the collection of the specimen. Specimens were produced with masturbation, either in a collection room at the fertility clinic or at the patient's home. To be collected at home, the sample had to be delivered within thirty minutes of production while being kept warm (i.e., placement of the receptacle in an axilla).

Freshly ejaculated sperm was allowed to liquefy before semen analysis. Liquefied semen was thoroughly mixed before an aliquot was placed on a standard count slide (Leja Products BV, Nieuw-Vennep, the Netherlands) for the preprocessing analysis. The slide was placed on a $37^{\circ} \mathrm{C}$ stage of an IVOS computer-assisted semen analyzer (Hamilton Thorn Biosciences, Beverly, MA). At least three random fields were evaluated for each analysis. Intra and inter-assay coefficients of variation of the parameters were less than $10 \%$ in all cases, pre- and post-processing.

Following the initial semen analysis, the sample was processed by first placing up to $4 \mathrm{~mL}$ of raw semen on a differential density gradient column consisting of $1 \mathrm{~mL}$ of $40 \%$ PureSperm and $1 \mathrm{~mL}$ 
of $80 \%$ PureSperm (Nidacon, Molndol, Sweden). The gradient was centrifuged for 20 minutes at $350 \times$ g, and subsequently, the $40 \%$ layer and the seminal plasma fraction were removed from the test tube, leaving the $80 \%$ layer undisturbed. Approximately 6-8 mL of sperm-washing medium and 5\% HAS (Cooper-Sage, Trumbull, CT) was added to the $80 \%$ layer and centrifuged for 10 minutes at $550 \times$ g. The sperm pellet was then reconstituted to approximately $0.5 \mathrm{~mL}$. The analysis of an aliquot of the processed sample was performed as previously described using the IVOS computer-assisted semen analyzer.

IUI and beta-human chorionic gonadotropin ( $\beta$-hCG) assay: IUI was performed approximately 24-hours ( $+/-3$ hours) after detection of a spontaneous urinary LH surge, or 36-hours (+/- 1 hours) after 10.000 IU $\beta$-hCG injection (Pregnyl, Merck, West Orange, NJ), (Novarel, Ferring Pharmaceuticals, Inc., Tarrytown, NY) or 250 mcg Ovidrel injection, (Merck-Serono Laboratories, Rockland, MD). hCG was administered when a transvaginal ultrasound revealed the largest follicle had a mean diameter of $\geq 18 \mathrm{~mm}$. The insemination was performed in a sterile fashion, using a flexible plastic catheter with the patient in the dorsal lithotomy position. The patient remained supine for at least ten minutes after the end of the insemination.

Serum $\beta$-hCG levels were analyzed 15 to 17 days after IUI to determine pregnancy status. Blood samples were assayed on an Immulite 2500 (Diagnostic Products Corporation, Los Angeles, CA) for a quantitative measurement of $\beta$-hCG. The Immulite uses a solid-phase two-site chemiluminescent immunometric assay with a sensitivity of $1 \mathrm{mIU} / \mathrm{mL}$ and a calibrated range to $5000 \mathrm{mIU} / \mathrm{mL}$. Intra- and inter-assay coefficients of variation were each less than $7 \%$. Most normal singleton pregnancies have levels in the range of 50 to $100 \mathrm{mIU} / \mathrm{mL}$ at this gestation. However, a level higher than five $\mathrm{mIU} / \mathrm{mL}$ was considered positive for pregnancy.

\section{Statistical analysis}

All statistical analyses were performed using the Statistical Package for the Social Sciences 11.0 (SPSS, Inc., Chicago, IL). Continuous variables were evaluated for normal distribution using the Kolmogorov-Smirnov test. Any variables that were not normally distributed were logarithmically transformed to obtain normality. Results are reported as mean value \pm standard deviation (SD). Categorical variables were evaluated with likelihood ratios. Likelihood ratios were calculated as:

$$
\mathrm{LR}+=\frac{\text { sensitivity }}{1-\text { specificity }}
$$

which is equivalent to;

$$
\mathrm{LR}+=\frac{\operatorname{Pr}(\mathrm{T}+/ \mathrm{D}+)}{\operatorname{Pr}(\mathrm{T}+/ \mathrm{D}-)}
$$

or "the probability of a person who falls into a grouping of the semen analysis having a pregnancy divided by the probability of a person who does not fall into the semen grouping having a pregnancy." Here " $\mathrm{T}+$ " or " $\mathrm{T}-$ " denotes that the classification into the semen analysis grouping is positive or negative, respectively. Likewise, "D+" or "D-" denotes that the pregnancy is present or absent, respectively. T-tests were used to compare for continuous variables. Levine's test for equality of variances was used to determine which $\mathrm{p}$ value to accept. Significance was taken as a $\mathrm{p} \leq 0.05$.

\section{Ethical approval}

The university's Human Subjects Research Ethics Committee approved this study (IRB number 95940). The authors have no conflict of interest.

\section{Results}

Baseline data of the cohort are provided in Table 1. An initial comparison without controlling for other semen analysis results was made to determine any single abnormal factor that gave lower pregnancy rates. Those with and without a pregnancy were classified based on volume $<1.5 \mathrm{~mL}$ or not, concentration $<15 \mathrm{mil} / \mathrm{mL}$ or not, $<32 \%$ forward motility or not, and $<39$ million sperm in the specimen. The results are presented in Table 2. Data are presented as mean values and SDs in the pregnant and not pregnant groups. The p-values for the likelihood ratio (one-sided, because it was hypothesized that abnormal results would have lower pregnancy values) are also presented comparing pregnancy rates in the groups that were normal or abnormal for the given parameter. As expected, the parameters were significantly different when comparing those grouped based on a parameter being abnormal or not. Among the parameters, only total sperm in specimens with $<39$ million gave lower pregnancy rates.

Next, semen analysis results were categorized based on the presence of one or more abnormal parameters, and precisely what parameters were abnormal. This gave the ability to control for confounding effects. At this stage, comparison was performed using volume (less than or greater than 1.5 $\mathrm{mL}$ ), concentration (less than or greater than $15 \mathrm{mil} / \mathrm{mL}$ ), and forward motility (less than or greater than $32 \%$ ). For this comparison, it was elected to exclude total sperm count

Table 1. The baseline characteristics of subjects

\begin{tabular}{|l|l|}
\hline & $\mathbf{n = 9 8 1}$ \\
\hline Maternal age (Years) & $37 \pm 6$ \\
\hline Duration of infertility (Years) & $24 \pm 14$ \\
\hline Previous pregnancies & $1.2 \pm 1.2$ \\
\hline Number of mature follicles & $2.4 \pm 1.3$ \\
\hline Maximum day 3 serum FSH (IU/L) & $7.9 \pm 2.4$ \\
\hline FSH: Follicle-stimulating hormone \\
\hline
\end{tabular}


because this value is not traditionally presented in a standard semen analysis reports. The results are presented in Table 3. Pregnancy rates are shown comparing all parameters in the normal group. As can be noted, none of the parameters or combination of these parameters predicted lower pregnancy rates when compared with normal specimens. Although two of the groups comprised few patients, given the trends in the total results, it is unlikely that the small numbers were the cause of lack of significance. The semen parameters for these seven groupings are presented in Table 4 for patients with and without pregnancies.

We made a comparison using total sperm count of less than or at least 39 million as well as volume and motility as predictors of pregnancy when compared with the normal group for all 3 because total sperm count in the specimen was the only factor that seemed to be associated with pregnancy rates. These results are shown in Table 5 . There are fewer comparisons performed than in Table 3 because we did not repeat any comparisons already presented. Consideration of sperm concentration was not performed. It should be noted than only the groups with total counts less than 39 million, motility less than $32 \%$, and volume less than $1.5 \mathrm{~mL}$ had a lower pregnancy rate. Even the group with low total count and motility but normal volume was not associated with pregnancy outcome, even though this group's results were equivalent to a low TMSC by the 2010 WHO parameters. Table 6 presents the semen analysis parameters from this group.

\section{Discussion}

Semen analysis has been the subject of debate for many years. It is unclear whether applying parameters found in a fertile population to an infertile population is valid $(13,14,32)$. However, to this day, semen analysis remains the primary objective measure of male factor infertility. For this reason, this study was performed to determine the relationship between abnormal semen parameters and pregnancy rates in couples undergoing IUI. Our study is the first of its kind, making it unique in nature, using the 2010 WHO parameters.

Table 2. Comparisons of pregnancy rates and parameters in the groups abnormal for any of the listed criteria according to the 2010 World Health Organization semen analysis criteria without controlling for other semen analysis parameters

\begin{tabular}{|c|c|c|c|c|}
\hline Parameters & $\begin{array}{l}\text { Abnormal WHO } \\
\text { parameter }\end{array}$ & $\begin{array}{l}\text { Normal WHO } \\
\text { parameter }\end{array}$ & $\begin{array}{l}\text { p (comparing abnormal and } \\
\text { normal WHO levels) }\end{array}$ & $\begin{array}{l}\text { Likelihood ratio (for } \\
\text { pregnancy) } p \text { value }\end{array}$ \\
\hline Volume (mL) & $\begin{array}{l}0.9 \pm 0.3 \\
(n=306)\end{array}$ & $\begin{array}{l}3.2 \pm 1.5 \\
(n=1925)\end{array}$ & $\leq 0.0001$ & 0.28 \\
\hline Concentration (million/mL) & $\begin{array}{l}9.8 \pm 3.5 \\
(n=250)\end{array}$ & $\begin{array}{l}59.0 \pm 42.4 \\
(n=1991)\end{array}$ & $\leq 0.0001$ & 0.11 \\
\hline Motility (\%) & $\begin{array}{l}19.3 \pm 8.2 \\
(\mathrm{n}=570)\end{array}$ & $\begin{array}{l}58.1 \pm 15.3 \\
(\mathrm{n}=1661)\end{array}$ & $\leq 0.0001$ & 0.11 \\
\hline $\begin{array}{l}\text { Total sperm count } \\
\text { (millions) }\end{array}$ & $\begin{array}{l}24.1 \pm 10.0 \\
(n=364)\end{array}$ & $\begin{array}{l}177.2 \pm 142.3 \\
(n=1867)\end{array}$ & $\leq 0.0001$ & 0.04 \\
\hline
\end{tabular}

Table 3. A comparison of data and pregnancy rates when one or more of the traditionally reported semen parameters are abnormal

\begin{tabular}{|c|c|c|c|}
\hline Parameters & Pregnancy rate & $\mathbf{n}$ & Pregnancy rate $p$ value \\
\hline All 3 normal & $20.9 \%$ & 1384 & - \\
\hline Low volume others normal & $24.8 \%$ & 170 & 0.24 \\
\hline Low concentration others normal & $17.4 \%$ & 99 & 0.39 \\
\hline Low motility others normal & $19.2 \%$ & 316 & 0.48 \\
\hline Low volume and low concentration, motility normal & $13.2 \%$ & 8 & 0.54 \\
\hline Low volume and low motility, concentration normal & $15.9 \%$ & 55 & 0.42 \\
\hline Low motility and low concentration, volume normal & $18.8 \%$ & 180 & 0.56 \\
\hline All 3 abnormal & $11.9 \%$ & 17 & 0.33 \\
\hline
\end{tabular}


The results demonstrate that if a single parameter is abnormal among those traditionally used to evaluate semen analysis, then pregnancy rates are unaffected with the exclusion of total sperm count less than 39 million in the specimen (Table 2). The data would be stronger if abnormal Kruger-Tyberg strict morphology data were available. However, because these patients are treated with IVF and ICSI at the center, conclusions cannot be drawn related to morphology. It remains important to note that the total quantity of sperm in the specimen affects pregnancy rates while other factors do not. Furthermore, pregnancy rates remain acceptable at $16 \%(p=0.4$, Table 5$)$.

Studies have found that TMSC was among the most important predictive factors of successful pregnancy rates (12,15-22). In Table 5, an evaluation of the parameters used to calculate
TMSC is presented. When the total count and motility were low (which equates with a measure of low TMSC), pregnancy rates were $18 \%$ and remained unaffected when compared with the normal group. This likely occurred because if measured then the TMSC would be abnormal once the level was below 12.48 million sperm. Most of the studies listed above only found decreased pregnancy rates when the TMSC was less than 10 million $(17-19,21)$ or 5 million $(15,16,20,22)$, which is well below the normal parameters quoted in the 2010 WHO guidelines. The value of TMSC in IUI nevertheless remains debated. Khalil et al. (16) in a retrospective study found that a TMSC of 5 million or higher was associated with higher pregnancy rates. In a descriptive retrospective cohort study by Kleppe et al. (23) based on 895 cycles in 273 couples, the cumulative pregnancy

Table 4. Preprocessing semen characteristics on the day of intrauterine insemination for the 7 different groupings

\begin{tabular}{|l|l|l|l|l|}
\hline Parameters & Volume (mL) & $\begin{array}{l}\text { Concentration } \\
\text { (millions/mL) }\end{array}$ & Motility (\%) & $\begin{array}{l}\text { Total motile sperm } \\
\text { count (million) }\end{array}$ \\
\hline All 3 normal & $3.1 \pm 1.4$ & $66.4 \pm 43.3$ & $59.4 \pm 15.0$ & $122.3 \pm 111.2$ \\
\hline Low volume others normal & $0.9 \pm 0.2$ & $70.3 \pm 40.0$ & $57.2 \pm 15.1$ & $36.2 \pm 34.3$ \\
\hline Low concentration others normal & $3.8 \pm 1.6$ & $10.8 \pm 3.0$ & $44.2 \pm 10.3$ & $18.1 \pm 10.9$ \\
\hline Low motility others normal & $3.3 \pm 1.6$ & $31.2 \pm 18.4$ & $20.2 \pm 8.0$ & $20.8 \pm 35.2$ \\
\hline Low volume and low concentration, motility normal & $1.0 \pm 0.2$ & $7.8 \pm 4.3$ & $49.0 \pm 13.9$ & $3.8 \pm 2.2$ \\
\hline Low volume and low motility, concentration normal & $0.9 \pm 0.3$ & $37.2 \pm 18.9$ & $18.2 \pm 9.1$ & $6.1 \pm 5.9$ \\
\hline Low motility and low concentration, volume normal & $3.5 \pm 2.2$ & $9.6 \pm 3.6$ & $17.3 \pm 7.9$ & $5.8 \pm 5.6$ \\
\hline All 3 abnormal & $0.8 \pm 0.2$ & $8.6 \pm 3.2$ & $17.8 \pm 8.7$ & $1.1 \pm 1.1$ \\
\hline Mean \pm standard deviation. The reference is the 2010 World Health Organization semen analysis normal parameter recommendations. \\
\hline
\end{tabular}

Table 5. Comparison of pregnancy rates based on total sperm count in specimen, volume and motility

\begin{tabular}{|l|l|l|}
\hline Parameters & Pregnancy rate & $\mathbf{n}$ \\
\hline All 3 normal & $21 \%$ & $\mathbf{p}$ value \\
\hline Low total sperm others normal & $16 \%$ & - \\
\hline Low total sperm and volume, motility normal & $23 \%$ & 81 \\
\hline Low total sperm and motility, volume normal & $18 \%$ & 66 \\
\hline Low total sperm, motility and volume & $11 \%$ & 0.29 \\
\hline $\begin{array}{l}\text { Pregnancy rate is compared with the groups with all 3 parameters normal for the calculation of the p value. P value is two sided } \\
\text { The reference is the 2010 World Health Organization semen analysis normal parameter recommendations. Statistically significant differences are in bold. }\end{array}$ & 0.34 \\
\hline
\end{tabular}

Table 6. Preprocessing semen characteristics on the day of intra uterine inseminations for the different groups based on total sperm count in the specimen

\begin{tabular}{|l|l|l|l|l|}
\hline Parameters & Volume (mL) & $\begin{array}{l}\text { Concentration } \\
\text { (millions/mL) }\end{array}$ & Motility (\%) & $\begin{array}{l}\text { Total motile sperm } \\
\text { count (million) }\end{array}$ \\
\hline All 3 normal & $3.2 \pm 1.4$ & $65.2 \pm 43.1$ & $59.2 \pm 15.1$ & $123.5 \pm 110.0$ \\
\hline Low total sperm others normal & $2.2 \pm 0.8$ & $14.0 \pm 6.0$ & $43.2 \pm 10.3$ & $13.3 \pm 5.1$ \\
\hline Low total sperm and volume, motility normal & $0.8 \pm 0.3$ & $32.3 \pm 17.0$ & $50.1 \pm 12.3$ & $12.9 \pm 6.2$ \\
\hline Low total sperm and motility, volume normal & $2.6 \pm 1.0$ & $10.9 \pm 4.9$ & $16.8 \pm 8.1$ & $4.8 \pm 3.3$ \\
\hline Low total sperm, motility and volume & $0.8 \pm 0.3$ & $23.3 \pm 14.1$ & $16.9 \pm 8.0$ & $3.2 \pm 2.3$ \\
\hline Mean \pm standard deviation. The reference is the 2010 World Health Organization semen analysis normal parameter recommendations. \\
\hline
\end{tabular}


rates increased from $17.3 \%$ as opposed to $25.5 \%$ with TMSC less than 1 million and greater than 1 million. Clearly, a 17\% pregnancy rate with a TMSC under a million remains an acceptable percentage. Pasqualotto et al. (24) concluded that the live birth rate increased with increased TMSC. However, they commented on the fact that success from IUI was mainly related to the percentage of motile sperm (24).

Typically, moderate male factor infertility is considered present when more than a single factor is abnormal $(13,25,26)$ and therefore, one would expect to see decreased pregnancy rates in this situation. However, our results show similar pregnancy rates when one, two, and in most cases three parameters were abnormal. The exception occurred if the total count was less than 39 million sperm, the volume was less than $1.5 \mathrm{~mL}$, and the forward motility was less than $32 \%$, in which case pregnancy rates decreased significantly, although remaining acceptable. Therefore, a couple with mild-to-moderate male factor infertility should be offered IUI as we would expect similar pregnancy rates as quoted in the literature of $13-20 \%$ $(6,23,27)$. Pregnancy rates in this study ranged from $11-25 \%$, excluding those that had a total sperm count less than 39 million, plus volume and forward motility also being abnormal. These pregnancy rates are evidently acceptable. Therefore, these rates play an important role in counseling couples when they present for assistive reproductive technology treatments.

There is significant debate as to whether sperm concentration affects pregnancy rates. The literature suggests a direct relationship with the number of spermatozoa in the specimen and pregnancy rates $(20,28)$. However, the results of the present study demonstrate that when sperm concentration is the single abnormal parameter, pregnancy rates (17\%) are excellent. Dorjpurev et al. (19) found slightly lower pregnancy rates per cycle when comparing sperm concentration of $<20 \times$ $106 / \mathrm{mL}(4.1 \%)$ vs $\geq 20 \times 10^{6} / \mathrm{mL}$ (7.3\%). In a prospective study, Haim et al. (29) showed that there was no significant difference in pregnancy rates with increasing sperm concentration. Pregnancy rates were $7.5 \%$ with concentration $<10 \times 10^{6 /}$ $\mathrm{mL}$, whereas they were $10.9 \%$ when concentrations were $>40 \times 10^{6} / \mathrm{mL}$ (29). Therefore, sperm concentration does not impact pregnancy rates significantly and, rather, TMSC is more predictive of successful IUI cycle.

A parameter that was not considered and poses a limitation to this study was sperm morphology. The majority of studies have consistently shown that sperm morphology is one of the best predictors of IVF and IUI outcomes (6). Coetzee et al. (30) demonstrated through a literature review that overall fertilization rates were $59.3 \%$ when morphology was <4\% and $77.6 \%$ when $>4 \%$ and pregnancy rates were $15.2 \%$ and $26.0 \%$, respectively. A literature review by Van Waart et al. (6) concluded that the tendency to become pregnant when sperm morphology was $\leq 4 \%$ was significantly decreased, and this was further supported by a review conducted by van der Merwe et al. (31) who concluded that morphology was the best predictor of sub-fertility and that a cut-off of $<5 \%$ should be used. However, sperm morphology is not traditionally calculated on the day of IUI because to do so would require killing a significant part of the specimen. It should also be noted that this population had a strict morphology on a recent semen analysis $\geq 4 \%$, which places them in the WHO normal range.

Another weakness of the study was the small number of subjects in particular groupings of semen parameters. Although these small numbers make it hard to conclude about the grouping individually, consistencies in the data as a whole are visible, notably the lack of differences. Nevertheless, confirmation of the results based on an even larger study would be helpful.

Data were purposefully not presented on female parameters or the stimulation protocol used. Slightly less than $2 \%$ used natural cycle IUI. The remaining patients used clomiphene, letrozole or gonadotropins. These data were not provided because it most closely resembles patient counseling on the day of IUI, based on the sperm. The physician cannot interpret the interplay of maternal age, body mass index, years of infertility and stimulation protocol, combined with semen analysis parameters. The physician instead states the sperm parameters and as such whether pregnancy rates are normal or diminished. This study permits an evidence-based interpretation of these parameters on the day of IUI, for the first time. It should be noted that one of the factors that affect pregnancy rates obtained with IUI cycles include stimulation medications. Pregnancy rates are often lower with oral drugs and higher with gonadotropins. In theory, the non-inclusion of these parameters represent a weakness of this study. However, by maintaining the premise that physicians counsel patients based only on semen parameters on the day of IUI, stimulation medications are not taken into consideration and as such were not included in the analysis.

Concurrently, clinical pregnancy rates are not presented because they are affected by factors that do not necessarily affect the pregnancy rate, i.e., sperm DNA fragmentation, history of recurrent pregnancy loss, uterine anomalies, and endometrial quality, among other factors (33-35). Lastly, this is not an examination of multiple pregnancies in IUI cycles, just the likelihood of pregnancy, based on semen parameters on IUI day. To evaluate the effect of semen parameters on multiple pregnancy rates with IUI is an interesting study; however, do to this study would require significant space and is worthy of its own paper.

One question that arises is whether biochemical pregnancy or clinical pregnancy should be used to measure semen 
parameter-related success. In our study, pregnancy rates were determined using serum $\beta$-hCG results, rather than with evidence of clinical pregnancy or live birth. However, it can be countered that semen capability is best measured in fertilization and biochemical pregnancy, whereas clinical pregnancy or live birth depends more on uterine environment, maternal age, and embryo developmental capacity. All these factors are sperm independent.

In conclusion, IUI remains an effective treatment when faced with a couple with male factor infertility. In all situations, pregnancy rates were at least $11 \%$ per cycle and therefore, certain abnormal semen analysis parameters should not be used to discourage IUI. Total sperm in the specimen and not the concentration of sperm per milliliter was the essential factor for predicting pregnancy. Therefore, a reorganization of the semen analysis report should be made emphasizing the total amount of sperm present and de-emphasizing the concentration of sperm.

Ethics Committee Approval: Stanford University ethics committee approval was obtained.

Informed Consent: Being a retrospective study informed consent was not required per the IRB protocol.

Peer-review: Externally peer-reviewed.

Author Contributions: Concept - C.A., A.K., M.H.D.; Data Collection or Processing - C.A., M.H.D.; Analysis or Interpretation - A.K., M.H.D.; Writer - A.K., M.H.D.

Conflict of Interest: No conflict of interest is declared by the authors.

Financial Disclosure: NIH grant 5K12HD01249 and the Stanford University Medical Scholars Program.

\section{References}

1. de Melo-Martin I. On cloning human beings. Bioethics 2002; 16: 246-65.

2. Grunewald S, Paasch U. Basic diagnostics in andrology. J Dtsch Dermatol Ges 2013; 11: 799-814.

3. Babayev SN, Park CW, Bukulmez O. Intracytoplasmic sperm injection indications: how rigorous? Semin Reprod Med 2014; 32 283-90.

4. Tournaye H. Male factor infertility and ART. Asian J Androl 2012; 14: 103-8.

5. Ombelet W, Wouters E, Boels L, Cox A, Janssen M, Spiessens C, et al. Sperm morphology assessment: diagnostic potential and comparative analysis of strict or WHO criteria in a fertile and a subfertile population. Int J Androl 1997; 20: 367-72.

6. Van Waart J, Kruger TF, Lombard CJ, Ombelet W. Predictive value of normal sperm morphology in intrauterine insemination (IUI): a structured literature review. Hum Reprod Update 2001; 7: 495-500.
7. Redmon JB, Thomas W, Ma W, Drobnis EZ, Sparks A, Wang C, et al. Semen parameters in fertile US men: the Study for Future Families. Andrology 2013; 1: 806-14.

8. Keegan BR, Barton S, Sanchez X, Berkeley AS, Krey LC, Grifo J. Isolated teratozoospermia does not affect in vitro fertilization outcome and is not an indication for intracytoplasmic sperm injection. Fertil Steril 2007; 88: 1583-8.

9. Catanzariti F, Cantoro U, Lacetera V, Muzzonigro G, Polito M. Comparison between WHO (World Health Organization) 2010 and WHO 1999 parameters for semen analysis - interpretation of 529 consecutive samples. Arch Ital Urol Androl 2013; 85: 125-9.

10. Ombelet W, Dhont N, Thijssen A, Bosmans E, Kruger T. Semen quality and prediction of IUI success in male subfertility: a systematic review. Reprod Biomed Online 2014; 28: 300-9.

11. Yavuz A, Demirci O, Sözen H, Uludoğan M. Predictive factors influencing pregnancy rates after intrauterine insemination. Iran J Reprod Med 2013; 11: 227-34.

12. Speyer BE, Abramov B, Saab W, Doshi A, Sarna U, Harper JC, et al. Factors influencing the outcome of intrauterine insemination (IUI): age, clinical variables and significant thresholds. J Obstet Gynaecol 2013; 33: 697-700.

13. Murray KS, James A, McGeady JB, Reed ML, Kuang WW, Nangia AK. The effect of the new 2010 World Health Organization criteria for semen analyses on male infertility. Fertil Steril 2012; 98: 1428-31.

14. Cooper TG, Noonan E, von Eckardstein S, Auger J, Baker HW, Behre $\mathrm{HM}$, et al. World Health Organization reference values for human semen characteristics. Hum Reprod Update 2010; 16: 231-45.

15. van Weert JM, Repping S, Van Voorhis BJ, van der Veen F, Bossuyt PM, Mol BW. Performance of the postwash total motile sperm count as a predictor of pregnancy at the time of intrauterine insemination: a meta-analysis. Fertil Steril 2004; 82: 612-20.

16. Khalil MR, Rasmussen PE, Erb K, Laursen SB, Rex S, Westergaard LG. Homologous intrauterine insemination. An evaluation of prognostic factors based on a review of 2473 cycles. Acta Obstet Gynecol Scand 2001; 80: 74-81.

17. Berker B, Şükür YE, Kahraman K, Atabekoğlu CS, Sönmezer M, Özmen B, et al. Absence of rapid and linear progressive motile spermatozoa "grade A" in semen specimens: does it change intrauterine insemination outcomes? Urology 2012; 80: 1262-6.

18. Demir B, Dilbaz B, Cinar O, Karadag B, Tasci Y, Kocak M, et al. Factors affecting pregnancy outcome of intrauterine insemination cycles in couples with favourable female characteristics. J Obstet Gynaecol 2011; 31: 420-3.

19. Dorjpurev U, Kuwahara A, Yano Y, Taniguchi T, Yamamoto Y, Suto A, et al. Effect of semen characteristics on pregnancy rate following intrauterine insemination. J Med Invest 2011; 58: 127-33.

20. Merviel P, Heraud MH, Grenier N, Lourdel E, Sanguinet P, Copin $\mathrm{H}$. Predictive factors for pregnancy after intrauterine insemination (IUI): an analysis of 1038 cycles and a review of the literature. Fertil Steril 2010; 93: 79-88.

21. Akanji Tijani $\mathrm{H}$, Bhattacharya $\mathrm{S}$. The role of intrauterine insemination in male infertility. Hum Fertil (Camb) 2010; 13: 226-32.

22. Tomlinson M, Lewis S, Morroll D; British Fertility Society. Sperm quality and its relationship to natural and assisted conception: British Fertility Society guidelines for practice. Hum Fertil (Camb) 2013; 16: 175-93.

23. Kleppe M, van Hooff MH, Rhemrev JP. Effect of total motile sperm count in intra-uterine insemination on ongoing pregnancy rate. Andrologia 2014; 46: 1183-8.

24. Pasqualotto EB, Daitch JA, Hendin BN, Falcone T, Thomas AJ Jr, Nelson DR, et al. Relationship of total motile sperm count and percentage motile sperm to successful pregnancy rates following intrauterine insemination. J Assist Reprod Genet 1999; 16: 476-82. 
25. Leushuis E, van der Steeg JW, Steures P, Repping S, Bossuyt PM Mol BW, et al. Semen analysis and prediction of natural conception. Hum Reprod 2014; 29: 1360-7

26. Ombelet W, Bosmans E, Janssen M, Cox A, Vlasselaer J, Gyselaers $\mathrm{W}$, et al. Semen parameters in a fertile versus subfertile population: a need for change in the interpretation of semen testing. Hum Reprod 1997; 12: 987-93.

27. Kupka MS, Ferraretti AP, de Mouzon J, Erb K, D'Hooghe T, Castilla JA, et al. Assisted reproductive technology in Europe, 2010: results generated from European registers by ESHRE. Hum Reprod 2014; 29: 2099-113.

28. Aydin Y, Hassa H, Oge T, Tokgoz VY. Factors predictive of clinical pregnancy in the first intrauterine insemination cycle of 306 couples with favourable female patient characteristics. Hum Fertil (Camb) 2013; 16: 286-90.

29. Haim D, Leniaud L, Porcher R, Martin-Pont B, Wolf JP, Sifer C. [Prospective evaluation of the impact of sperm characteristics on the outcome of intra-uterine insemination]. Gynecol Obstet Fertil 2009; 37: 229-35.

30. Coetzee K, Kruge TF, Lombard CJ. Predictive value of normal sperm morphology: a structured literature review. Hum Reprod Update 1998; 4: 73-82.
31. van der Merwe FH, Kruger TF, Oehninger SC, Lombard CJ. The use of semen parameters to identify the subfertile male in the general population. Gynecol Obstet Invest 2005; 59: 86-91.

32. Papillon-Smith J, Baker SE, Agbo C, Dahan MH. Pregnancy rates with intrauterine insemination: comparing 1999 and 2010 World Health Organization semen analysis norms. Reprod Biomed Online 2015; 30: 392-400.

33. Luco SM, Agbo C, Behr B, Dahan MH. The evaluation of pre and post processing semen analysis parameters at the time of intrauterine insemination in couples diagnosed with male factor infertility and pregnancy rates based on stimulation agent. A retrospective cohort study. Eur J Obstet Gynecol Reprod Biol 2014; 179: 159-62.

34. Zini A. Are sperm chromatin and DNA defects relevant in the clinic? Syst Biol Reprod Med 2011; 57: 78-85.

35. Zeadna A, Son WY, Moon JH, Dahan MH. A comparison of biochemical pregnancy rates between women who underwent IVF and fertile controls who conceived spontaneously. Hum Reprod 2015; 30: 783-8. 